\title{
Robust, Wearable, On-body Antenna Relying on Half Mode Substrate Integrated Waveguide Techniques
}

\author{
Sam Agneessens, Hendrik Rogier \\ Department of Information Technology (INTEC) \\ Ghent University \\ Ghent, Belgium \\ sam.agneessens@intec.ugent.be
}

\author{
Riccardo Moro, Maurizio Bozzi \\ Department of Electronics \\ University of Pavia \\ Pavia, Italy
}

\begin{abstract}
A compact, robust, wearable antenna for body-worn applications in the $2.4 \mathrm{GHz}$ Industrial Medical and Scientific band is designed, fabricated and tested. This novel compact textile cavity backed slot antenna combines a half-mode substrate integrated waveguide topology with an additional row of shorting vias for miniaturization. Excellent free space performance is achieved with a measured $4.6 \%$ impedance bandwidth, maximal gain of $4.7 \mathbf{d B i}$ and radiation efficiency of $81.3 \%$. On-body measurements reveal minimal frequency detuning when the antenna is worn by a test subject as well as a negligible impedance bandwidth reduction to $4.5 \%$. The low calculated Specific Absorption Rate of $0.51 \mathrm{~W} / \mathrm{kg}$ averaged over $1 \mathrm{~g}$ of tissue demonstrates high antenna body isolation. Therefore, this design is an attractive option as antenna in smart textile systems.
\end{abstract}

\section{INTRODUCTION}

Textile antennas are attractive components to improve the integration of electronics into smart textiles, as they offer a flexible solution without performance loss and at a minimal hindrance to the user [1], [2]. But, as for all technologies, there remains room for improvement. For textile antennas, there still remain challenges to reduce their size without performance loss in real operating conditions. Indeed, extensive size reduction is limited by the need to shield the body from the antenna radiation. This is necessary to meet radiation safety regulations (Specific Absorption Rate, SAR) and to guarantee robust and stable performance in the presence of the human body [3]. To this end, a ground plane, which needs to be larger than the actual antenna, is typically used, resulting in a significant overall size.

Although the body offers quite a large surface for the integration of antennas, body-worn applications can certainly benefit from devices with small sizes and excellent on-body performance, as this opens up possibilities for array configurations, MIMO systems, etc. To solve this problem, we rely on a combination of different techniques: textile materials and a Substrate Integrated Waveguide (SIW) topology. Textile fabrics provide flexibility and wearability whereas SIW techniques exhibit waveguide-like performance in planar form [4]. Implementing textile antennas by means of SIW techniques enables us to develop flexible and compact designs with excellent on body performance.

\section{Antenna Design}

The goal is to design a compact wearable antenna, with good performance in the $2.4 \mathrm{GHz}$ ISM band and robust characteristics in close vicinity of the human body. These properties result in a component that is easily integrated into smart garments for rescue workers, medical personnel, law enforcement officers, etc.

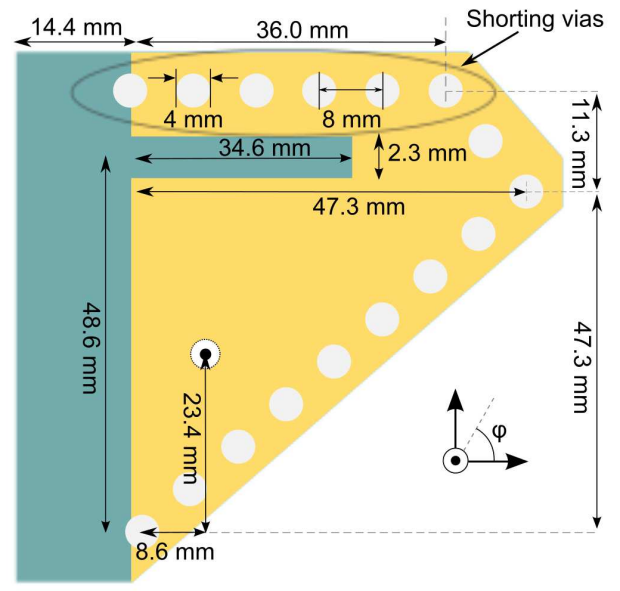

Fig. 1. Geometry and dimensions of the designed compact on body antenna.

The design relies on textile materials, an HMSIW topology, and a row of shorting vias to create a miniaturized wearable antenna. Starting point is a cavity backed slot antenna, operating at its first resonant mode, with the slot along the diagonal of the cavity. Thanks to the symmetry of the field distribution, a virtual magnetic wall can be implemented along the diagonal perpendicular to the slot. One half of the cavity can then be cut away without affecting the radiation performance, reducing the cavity size by $50 \%$. We now obtain a triangular cavity, with an open wall along its longest edge and a slot in the middle, which divides the structure in an upper and a lower cavity. A further size reduction is achieved by shorting the area above 
the slot with a row of vias, removing the upper cavity from the structure. This yields an antenna that is slightly larger than one quarter of the original cavity backed slot antennas, while still using a ground plane to provide excellent shielding between body and antenna. The final layout is shown in Fig. 1.

Optimization is conducted with CST microwave studio to fulfill the matching criterion in the desired frequency band: $|S(1,1)|<-10 d B$, between $2.4 \mathrm{GHz}$ and $2.4835 \mathrm{GHz}$ for an impedance level $Z_{0}=50 \Omega$. The antenna is fully fabricated using textile materials. A fire retardant foam with thickness $3.94 \mathrm{~mm}$, whose electrical properties were characterized as $\epsilon_{r}=1.495$ and $\tan \delta=0.016$, is applied as antenna substrate. Conductive planes are hand-cut from conductive Taffeta, a copper plated nylon fabric with surface resistivity $0.18 \Omega / s q$ @ $2.45 \mathrm{GHz}$. The vias are realized by means of brass eyelets, pierced though the antenna substrate by a hand press.

\section{Simulation AND MEASUREMENT RESUlts}

Simulations and measurements are conducted to verify the performance. In an anechoic chamber, the $3 \mathrm{D}$ radiation pattern and the reflection coefficient, are measured to evaluate the free space performance. On-body measurements are also conducted to test the antennas robustness when positioned on a test subject wearing a t-shirt.

Fig. 2. shows the simulated and measured reflection coefficient in free space and on a human body. Good agreement is observed between the simulated and the measured results in free space. The antenna covers the complete $2.4 \mathrm{GHz}$ ISM band, with a measured bandwidth of $4.6 \%$. The measured on-body reflection coefficient does not differ much from the free space result. An impedance bandwidth of $4.5 \%$ is measured, indicating that the antenna has very robust on body performance thanks to the excellent antenna-body isolation.

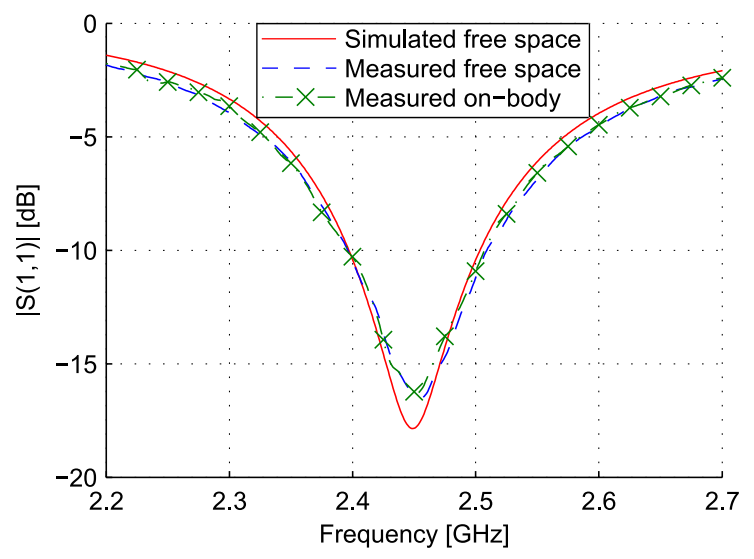

Fig. 2. Antenna performance in free space and on-body.

In Fig.3, in which the measured and simulated gain in the E- $\left(\phi=135^{\circ}\right)$ and H-plane $\left(\phi=45^{\circ}\right)$ are plotted. A good agreement between measurements and simulations is demonstrated. The maximum measured gain equals $4.7 \mathrm{dBi}$, which is close to the simulated value of $4.9 \mathrm{dBi}$. Measurements reveal a radiation efficiency of $81.3 \%$, compared to $79.2 \%$ in simulation. The measured $3 \mathrm{~dB}$ beamwidth is $130^{\circ}$, whereas a measured front-to back lobe suppression is $14.7 \mathrm{~dB}$.

The amount of radiation absorbed by the body is calculated with CST. The calculated SAR, with body-antenna spacing $1 \mathrm{~mm}$, is $0.51 \mathrm{~W} / \mathrm{kg}$ averaged over $1 \mathrm{~g}$ of tissue with an 0.32 $\mathrm{W}$ input power (equal to $20 \mathrm{dBm}$ Effective Isotropic Radiated Power, EIRP, the maximum allowed level in this frequency band). This is well below the limit of $1.6 \mathrm{~W} / \mathrm{kg}$.

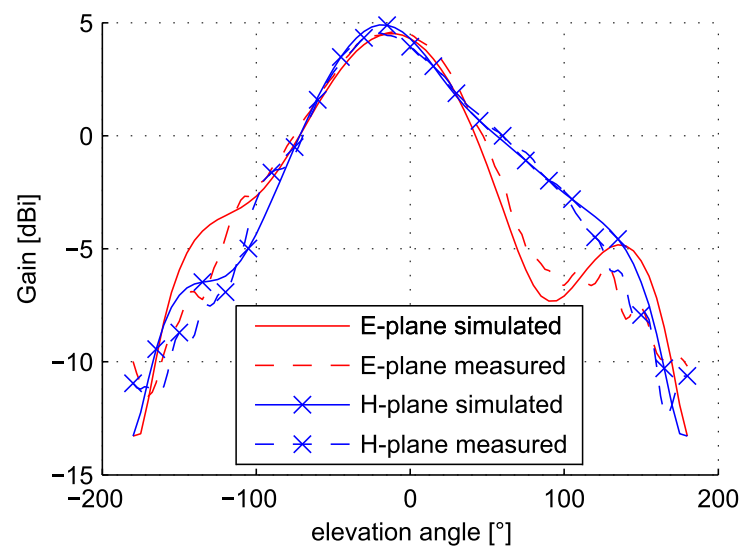

Fig. 3. Measured and simulated radiation pattern in the E-plane $\left(\phi=135^{\circ}\right)$.

\section{CONCLUSIONS}

A textile antenna for use in body worn applications in the $2.4 \mathrm{GHz}$ ISM band was presented. It features a HMSIW topology and uses an extra row of shorting vias to achieve a compact size. Excellent agreement is seen between simulations and measurements in terms of impedance matching and radiation performance. The design has a measured impedance bandwidth of $4.6 \%$, maximal gain of $4.7 \mathrm{dBi}$ and efficiency of $81.3 \%$. The measured reflection coefficient remains very stable in close vicinity of the body and the low calculated SAR value of $0.51 \mathrm{~W} / \mathrm{kg}$ indicates that there is good isolation between the body and the radiating antenna. This combination of flexible textile materials and the HMSIW technique has resulted in a wearable antenna that is compact, enhibits robust on-body behavior and is well suited for integration into smart textiles.

\section{ACKNOWLEDGEMENT}

Part of this work was supported by BELSPO through the IAP Phase VII BESTCOM project.

\section{REFERENCES}

[1] M. Klemm and G. Troester, "Textile UWB antennas for wireless body area networks," IEEE Transactions On Antennas and Propagation, vol. 54, no. 11, pp. 3192-3197, Nov. 2006.

[2] E. K. Kaivanto, M. Berg, E. Salonen, and P. de Maagt, "Wearable circularly polarized antenna for personal satellite communication and navigation," IEEE Transactions On Antennas and Propagation, vol. 59, no. 12, pp. 4490-4496, Dec. 2011.

[3] S. Z. Zhu and R. Langley, "Dual-band wearable textile antenna on an EBG substrate," IEEE Transactions On Antennas and Propagation, vol. 57, no. 4, pp. 926-935, Apr. 2009.

[4] T. Kaufmann and C. Fumeaux, "Wearable textile half-mode substrateintegrated cavity antenna using embroidered vias," pp. 1-1, 2013. 